\title{
A MATHEMATICAL MODEL FOR PRESSURE SWING ADSORPTION
}

\author{
D.J.G. Sebastian \\ BOC Limited, UK
}

\section{Introduction}

Pressure Swing Adsorption (PSA) as a physical method of separation of gas mixtures has rapidly gained importance. The incentive for a mathematical model comes from the fact that accuracy of prediction is essential for optimal plant design.

The PSA principle of gas separation is based on two fundamental facts :

1) Some substances adsorb some gases in preference to other gases.

2) The amount adsorbed for any given gas increases as the pressure of the gas increases.

As an illustration, consider the following two-step adsorption cycle to purify helium diving gas. Helium diving gas is principally a mixture of oxygen and helium. When the diving chambers are depressurised, the contaminated gas will consist mainly of carbon dioxide, oxygen and helium. Traditionally this waste gas has been thrown away. The PSA cycle described below is a method of purifying this gas and recovering expensive helium.

The adsorbent chosen has a strong preference for adsorbing all gases except helium.

Step 1. Purification

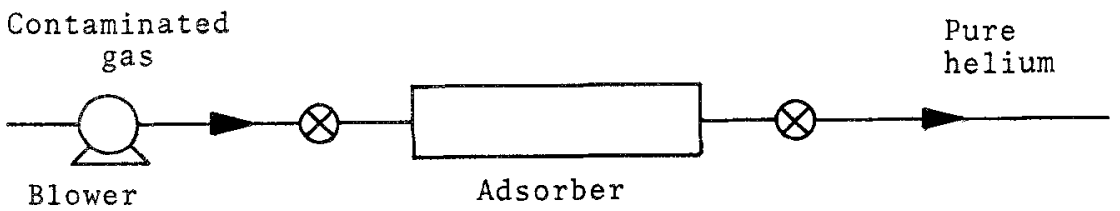


During the high pressure purification step oxygen and the contaminants are preferentially adsorbed and the output from the bed is pure helium. The bed will gradually become saturated, and when it is no longer able to purify the input gas, it is changed over to regeneration.

Step 2. Regeneration

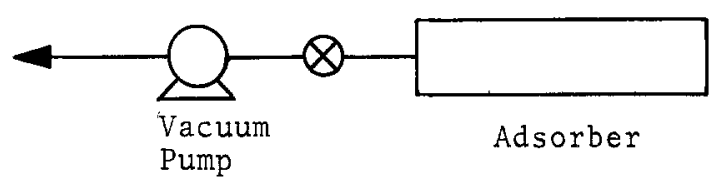

As the bed is evacuated its capacity to hold gases decreases, thus losing oxygen and the contaminants. At the end of the evacuation the bed is ready for purification again.

The term pressure swing comes from the fact that a swing in the pressure between the steps is essential for the process to function. In actual practice the cycles are, in general, more complex than the one described, but the principles of operation remain the same.

This paper describes a model which has been developed for simulating the evacuation step.

\section{Equations Forming the Model}

a) Partial Differential Equations for the Bed.

The equations themselves are based on standard chemical engineering principles and their derivation is not given here. The variables of the model are set out below:

Independent Variables

$$
\begin{aligned}
& x \text { - distance along the bed } \\
& \theta \text { - time }
\end{aligned}
$$

Dependent Variables

$$
\begin{aligned}
& u \text { - flow rate } \\
& v \text { - gas phase composition } \\
& w \text { - pressure }
\end{aligned}
$$


i) Overal1 Mass Balance Equation

$$
\frac{\partial u}{\partial x}+\left[\frac{a \varepsilon}{R T}+a \xi(v, w)\right] \frac{\partial v}{\partial \theta}+a \eta(v, w) \frac{\partial w}{\partial \theta}=0
$$

ii) Single Component Mass Balance Equation

$$
\frac{\partial V w}{\partial x}+\left[\frac{a \varepsilon}{R T}+a \gamma(v, w)\right] \frac{\partial v w}{\partial \theta}+a \delta(v, w) \frac{\partial w}{\partial \bar{\theta}}=0
$$

iii) Momentum Balance Equation

$$
w \frac{\partial w}{\partial x}+\alpha u+\beta u^{2}=0
$$

In the above equations $\alpha, \beta, a, \varepsilon, R$ and $T$ are constants.

b) The Initial and Boundary Conditions

$$
\begin{aligned}
& u(x, 0)=0 \\
& v(x, 0)=y_{0} \\
& w(x, 0)=p_{0}
\end{aligned}
$$

Two of the boundary conditions are at $x=1$ and are straightforward.

$$
\begin{aligned}
& u(1, \theta)=0 \\
& v(1, \theta)=y_{0}
\end{aligned}
$$$$
0 \leq x \leq 1
$$

The vacuum pump, pipework and fittings are incorporated into the model through the third boundary condition. This is specified at $x=0$ in the form of the nonlinear algebraic equation

$$
\phi(u(0, \theta), w(0, \theta))=0 \quad \theta>0
$$

This form of the boundary condition gives rise to two difficulties

i) Unlike the case, for example, of the linear diffusion equation, the boundary value problem cannot be solved explicitly. It is necessary to start with a first approximation for the solution and by successive iteration force it to satisfy boundary condition 2.6 . 
ii) The non-linearity of the boundary condition leads to multiplicity of solution.

As the fitting of the boundary condition is iterative, occasionally an unwanted solution is obtained. When this occurs it is usually in the form of switching from one solution to the other.

However, certain monotonicity conditions shown in the next section may be imposed on the solution to prevent switching to the unwanted solution.

c) Monotonicity Conditions

These conditions are derived from physical consideration

i) For $x_{1}>x_{2}$ and $\theta>0$,

$$
\begin{aligned}
& w\left(x_{2}, \theta\right)<w\left(x_{1}, \theta\right) \\
& u\left(x_{2}, \theta\right)<u\left(x_{1}, \theta\right)
\end{aligned}
$$

ii) For $\theta_{1}>\theta_{2}$,

$$
\mathrm{w}\left(1, \theta_{1}\right)<\mathrm{w}\left(1, \theta_{2}\right)
$$

In addition, for $\theta>0,0 \leq x<1$

$$
u(x, \theta)<0
$$

While the desired solution satisfies all the above conditions, the unwanted solution will violate at least one of them beginning with i). Thus the algorithm is given a test for detecting when there is a chance of switching to the unwanted solution.

The extreme importance of these conditions will become clearer when the technique of solution is described. Their use has been entirely successful in eliminating the unwanted solution and is thus sufficient to ensure uniqueness of solution. 
d) The Type of Equations

The three partial differential equations are first order quasilinear. They are hyperbolic-parabolic in the sense that there are only two distinct characteristics, and the third characteristic coincides with one of the first two. All the three characteristics are real and are given by

$$
\begin{aligned}
\frac{d x}{d \theta} & =\frac{u}{\left(\gamma^{\prime}-\xi^{\prime} v\right)} \\
\text { and } \quad & =0 \\
\text { where } & =0 \\
\gamma^{\prime} & =\left[\frac{a \varepsilon}{\mathrm{RT}}+\gamma(v, w)\right] w \\
\xi^{\prime} & =\frac{\mathrm{a} \varepsilon}{\mathrm{RT}}+\xi(v, w)
\end{aligned}
$$

This is useful in studying the propagation of discontinuities in the boundary conditions and numerical stability.

\section{The Method of Solution}

a) General Approach

The partial differential equations are replaced by finite difference equations which are consistent with them. The iterative procedure for fitting the boundary conditions is carried out with a one-variable minimization method starting with a first approximation. At $x=1$ boundary conditions fix the values of $u(1, \theta)$ and $v(1, \theta)$. Let $w^{*}(1, \theta)$ be the approximation for $w(1, \theta)$ which is as yet unknown. Using $u(1, \theta), v(1, \theta)$ and $w^{*}(1, \theta)$ the finite difference equations can be solved to give $u^{*}(0, \theta)$ and $w^{*}(0, \theta)$ as approximations for $u(0, \theta)$ and $w(0, \theta)$ respectively. When $W^{*}(I, \theta)$ is the correct value

$$
\phi\left(u^{*}(0, \theta), w^{*}(0, \theta)=0\right.
$$

This value is determined by minimizing $\phi^{2}\left(u^{*}(0, \theta), w^{*}(0, \theta)\right)$ with respect to $\mathrm{W}^{*}(1, \theta)$. The minimization method employed is successive quadratic fitting. 
b) The Finite Difference Scheme

The differential equations are retained in the first order, and the numerical scheme is explicit. A judicious choice of finite differences results in a simple scheme of solution. Some attractive looking schemes were intractable for stability analysis and were abandoned.

The final numerical scheme used is as follows.

$$
\begin{aligned}
& u_{i, j}=u_{i, j+1}+\operatorname{ar}\left\{\left(\frac{\varepsilon}{R T}+\xi\right)\left(v_{i, j+1}-v_{i-1, j+1}\right)+n\left(w_{i, j+1}-w_{i-1, j+1}\right)\right\} .3 .1 \\
& w_{i, j}=w_{i, j+1}-\delta x\left(\alpha u_{i, j}+\beta u_{i, j}^{2}\right) / w_{i-1, j+1} \\
& v_{i, j}=\frac{\left\{-u_{i, j} v_{i, j+1}+v_{i-1, j}\left(\frac{a \varepsilon}{R T}+a \gamma\right)\left(w_{i, j+1}+w_{i, j}-w_{i-1, j}\right)-a \delta\left(w_{i, j}-w_{i-1, j}\right)\right\}}{u_{i, j+1}-2 u_{i, j}+a\left(\frac{\varepsilon}{R T}+\gamma\right) w_{i, j+1}}
\end{aligned}
$$

where $\xi, \eta, \gamma$ and $\delta$ are calculated at the grid point $i-1, j$ and $r=\frac{\delta x}{\delta}$.

The notation used for the grids is

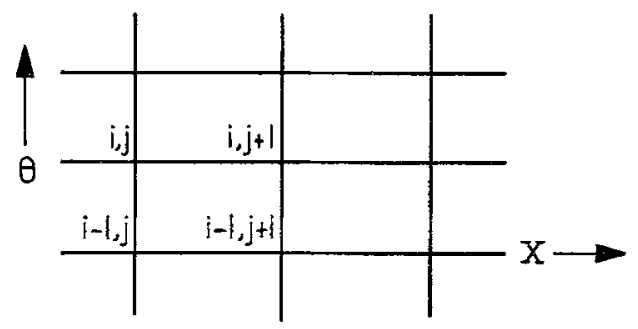

c) Consistency and Stability

It is easily verified that the finite difference equations are consistent with partial differential equations and the truncation errors are of the first order.

To study stability the error propagation equations are linearized. The eigen values characterizing the propagation of errors at time step $i$ are bounded by 
$\lambda_{i \max }=\max _{j} \mid \frac{\operatorname{ar}\left(\frac{\varepsilon}{\mathrm{RT}}+\gamma\right)\left(w_{i j}-2 w_{i-1, j}\right)}{u_{i, j+1}-u_{i, j}+\operatorname{ar}\left(\frac{\varepsilon}{\mathrm{RT}}+\gamma\right) w_{i, j+1}}$

As one would expect with nonlinear equations, $\lambda$ itself depends on the solution. It is therefore not possible to calculate $\lambda_{i} \max$ 'a priori'. Any bound one can put on $\lambda_{i \max }$ is also unrealistic.

However, stability analysis is invaluable in evaluating a method. Some numerical schemes were shown to be intrinsically unstable. Some other schemes were detected to be prone to instability, which was often revealed by a hand calculation.

Furthermore, the stability test enables the algorithm to detect it when stability is lost.

d) Multiplicity of Solution

What has been discussed so far is concerned with the conventional ideas of consistency, stability, characteristics and so on. One of the most unusual and interesting features of this problem is the fact that the solution is not unique. This usually manifested itself in the form of sudden switching from the desired solution to the other. The cause of this can be pinned down to the nonlinearity of the boundary condition,

$$
\phi(u(0, \theta), w(0, \theta))=0
$$

In order to eliminate the unwanted solution the following points must be noted:

i) The algorithm must be able to detect the unwanted solution. As mentioned earlier when the solution is not the desired one at least one of the monotonicity conditions will be violated and this can be built into the algorithm.

ii) The algorithm must suppress the tendency to move towards the unwanted solution. This is achieved by applying a penalty function during the minimization. 
Consider what happens at time $\theta_{i}$. Let $w^{(n)}\left(1, \theta_{i}\right)$ represent the $n$th approximation to $w\left(1, \theta_{i}\right)$. There exists $\bar{w}\left(1, \theta_{i}\right)$, such that whenever $w^{(n)}\left(1, \theta_{i}\right)>\bar{w}\left(1, \theta_{i}\right)$ the monotonicity conditions are violated and vice versa. The value of $\bar{w}\left(1, \theta_{i}\right)$ is itself unknown and so it is not possible to put an explicit constraint on $w^{(n)}\left(1, \theta_{i}\right)$. The only alternative is to apply a penalty function whenever a monotonicity condition is violated, which is done as follows:

If $\quad u^{(n)}\left(x_{i j}, \theta_{i}\right)<u^{(n)}\left(x_{i j+1}, \theta_{i}\right) \quad$ for $1 \leq j \leq n-1$

then no penalty is added.

$p\left(w^{(n)}\left(1, \theta_{i}\right)\right)= \begin{cases}10^{4}\left(1+\left(w^{(n)}\left(1, \theta_{i}\right)-w\left(1, \theta_{i-1}\right)\right)^{2}\right), & \text { if } w^{(n)}\left(1, \theta_{i}\right)>w\left(1, \theta_{i-1}\right) \\ 10^{4} \frac{1}{\left.1+(w)\left(1, \theta_{i}\right)-w\left(1, \theta_{i-1}\right)\right)^{2}}, \text { if } w^{(n)}\left(1, \theta_{i}\right) \leq w\left(1, \theta_{i-1}\right)\end{cases}$

The following graphs represent schematically the functions involved.

a) $\quad \phi^{2}\left(u^{*}\left(0, \theta_{i}\right), w^{*}\left(0, \theta_{i}\right)\right)$

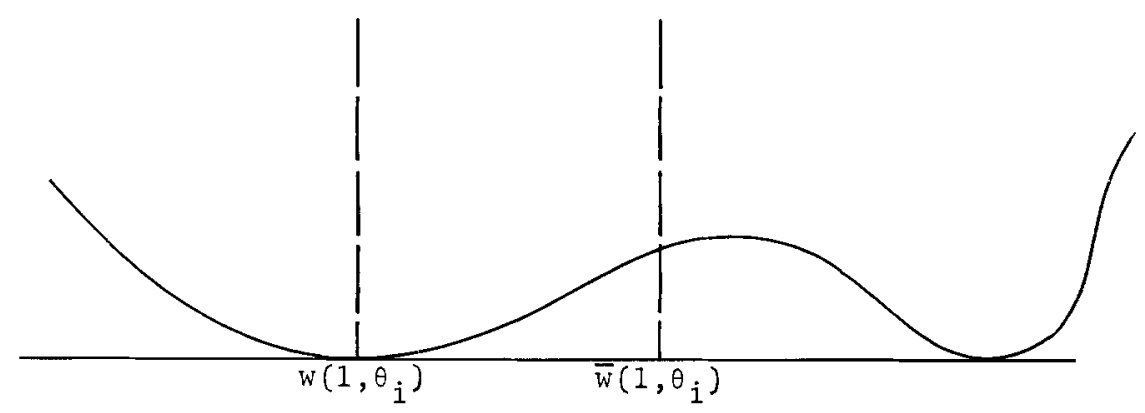

b) $\quad p\left(w^{(n)}\left(1, \theta_{i}\right)\right)$

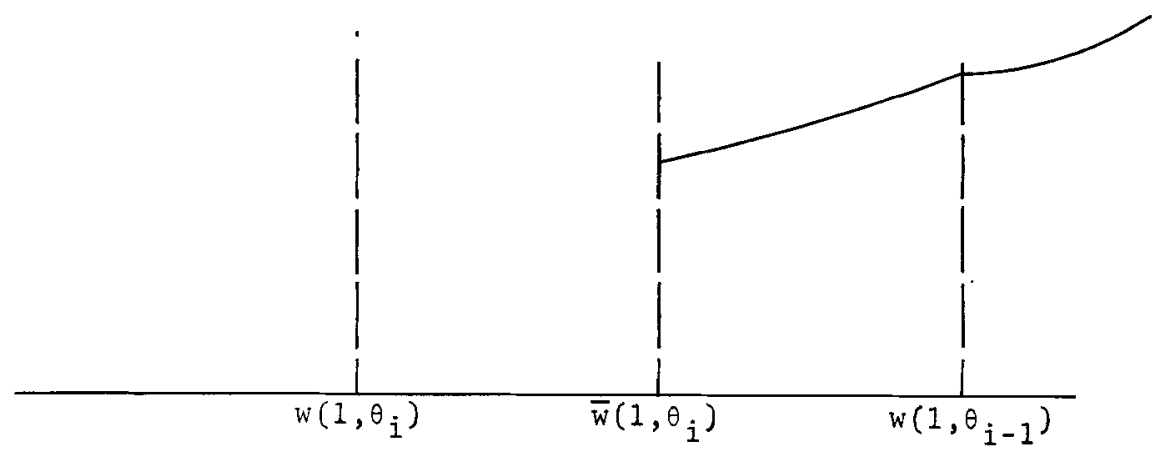


c) $\quad \phi^{2}\left(u^{*}\left(0, \theta_{i}\right), w^{*}\left(0, \theta_{i}\right)+p\left(w^{(n)}\left(1, \theta_{i}\right)\right)\right.$

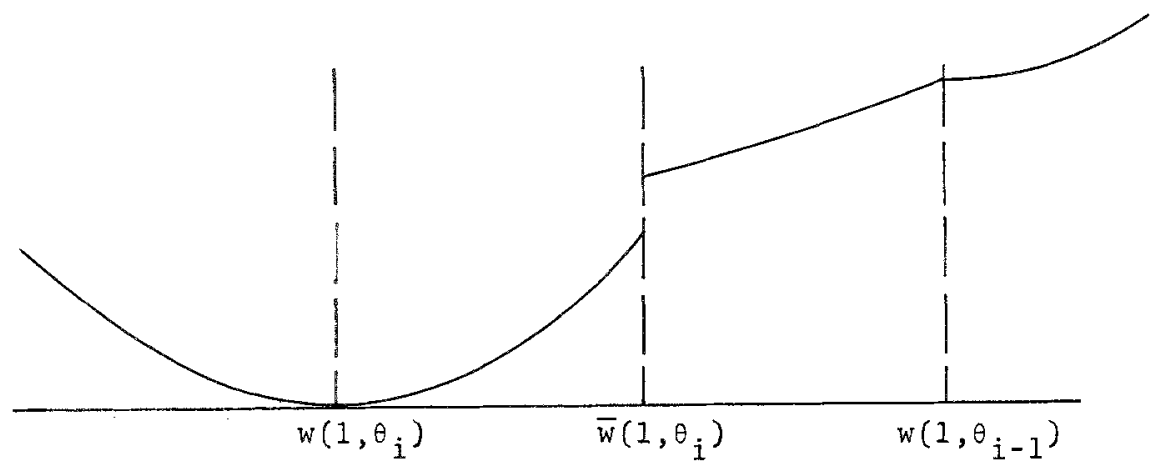

There is a discontinuity in the function to be minimized at $\overline{\mathrm{w}}\left(1, \theta_{i}\right)$ but this cannot be helped as $\bar{w}\left(1, \theta_{i}\right)$ is unknown. In any case, this gave rise to no difficulty with the minimization method.

\section{Simplification of the Model for Design Use}

For process evaluation one would like to calculate $u, v$ and $w$. On the other hand for design purposes one is more interested in $u$ and $w$ while $v$ is of little interest. Therefore, partial differential equation 2.2 is replaced by a simple algebraic equation

$$
v=v_{1}+\frac{\left(v_{2}-v_{1}\right)}{\left(w_{2}-w_{1}\right)}\left(w-w_{1}\right)
$$

Now that $v$ depends only on $w$ the system of differential equations reduces to

$$
\begin{aligned}
\frac{\partial u}{\partial x}+\zeta(w) \frac{\partial w}{\partial \theta} & =0 \\
w \frac{\partial w}{\partial x}+\alpha u+\beta u^{2} & =0
\end{aligned}
$$

The resulting finite difference scheme is much simpler.

$$
\begin{aligned}
& u_{i, j}=u_{i, j+1}+\operatorname{ar}\left\{\left(\frac{\varepsilon}{R T}+\xi\right) \frac{\left(v_{2}-v_{1}\right)}{\left(w_{2}-w_{1}\right)}+n\right\}\left(w_{i, j+1}-w_{i-1, j+1}\right) 4.4 \\
& w_{i, j}=w_{i, j+1}-\delta x\left(\alpha u_{i j}+\beta u_{i j}{ }^{2}\right) / w_{i-1, j}
\end{aligned}
$$


The eigen values characterizing the propagation of the errors are bounded at time step i by

$$
\lambda_{i \max }=\max _{j}\left|\frac{w_{i, j}-w_{i, j+1}}{w_{i-1, j}}\right|
$$

The rest of the procedure is exactly the same as in section 3 .

\section{Programming Details}

The first approximation for $w\left(1, \theta_{i}\right)$ is obtained by

$$
\mathrm{w}^{(1)}\left(1, \theta_{i}\right)=1.8 \mathrm{w}\left(1, \theta_{i-1}\right)-0.8 \mathrm{w}\left(1, \theta_{i-2}\right)
$$

The stopping criterion for the minimization procedure is

$$
\phi^{2}\left(u^{(n)}(0, \theta), w^{(n)}(0, \theta)\right) \leq \varepsilon^{\prime}
$$

The value of $\varepsilon^{\prime}$ is set by the program depending on the plant size and is of the order of $10^{-7}$. In general the one-variable minimization is rapid and the solution is found in about eight function evaluations. During the first time step, occasionally the number of function evaluations was as much as 20 , which is because equation 4.7 cannot be used to obtain a first approximation. The use of the penalty function has little effect on the performance, as it is not transgressed often.

\section{Results}

The results obtained are presented in a graphical form. The full model is used in studying how the composition varies along the length of the bed at a given time. The simplified model on the other hand is used for predicting how the pressure at the end of the bed and the vacuum pump suction pressure vary with time.

Graph 1 was obtained using the full model, while graphs 2, 3 and 4 were obtained using the simplified model. The simplified model was, in particular, very useful in plant design. This model has been tested in 3 stages: 
i) Simulating an existing plant - Graph 2 .

ii) Predicting the behaviour of a pilot plant and comparing it with observations - Graph 3.

iii) Designing a full scale plant and then testing the design against actual performance - Graph 4.

In each case the correspondence was exceptionally good and the technique has proved reliable and accurate in every instance.

\section{References}

1 Ames, W.F. (1969) Numerical Methods for Partial Differential Equations, London, Thomas Nelson and Sons.

2 Armond, J.W. and Smith, K.C. (1973) Adsorption as a Technique for Gas Separation, Cryotech 73 Proceedings.

3 Smith, G.D. (1971) Numerical Solution of Partial Differential Equations, London, Oxford University Press.

4 Smith, M.G. (1967) Theory of Partial Differential Equations, London, Van Nostrand

5 Webber, D.A. (1972) Adsorptive Removal of Carbon Dioxide from Air at Intermediate Low Temperatures, The Chemical Engineer.

Acknowledgements:

I would like to thank BOC Ltd for allowing me to present this paper and, in particular, Mr. K.C. Smith for his personal interest. 


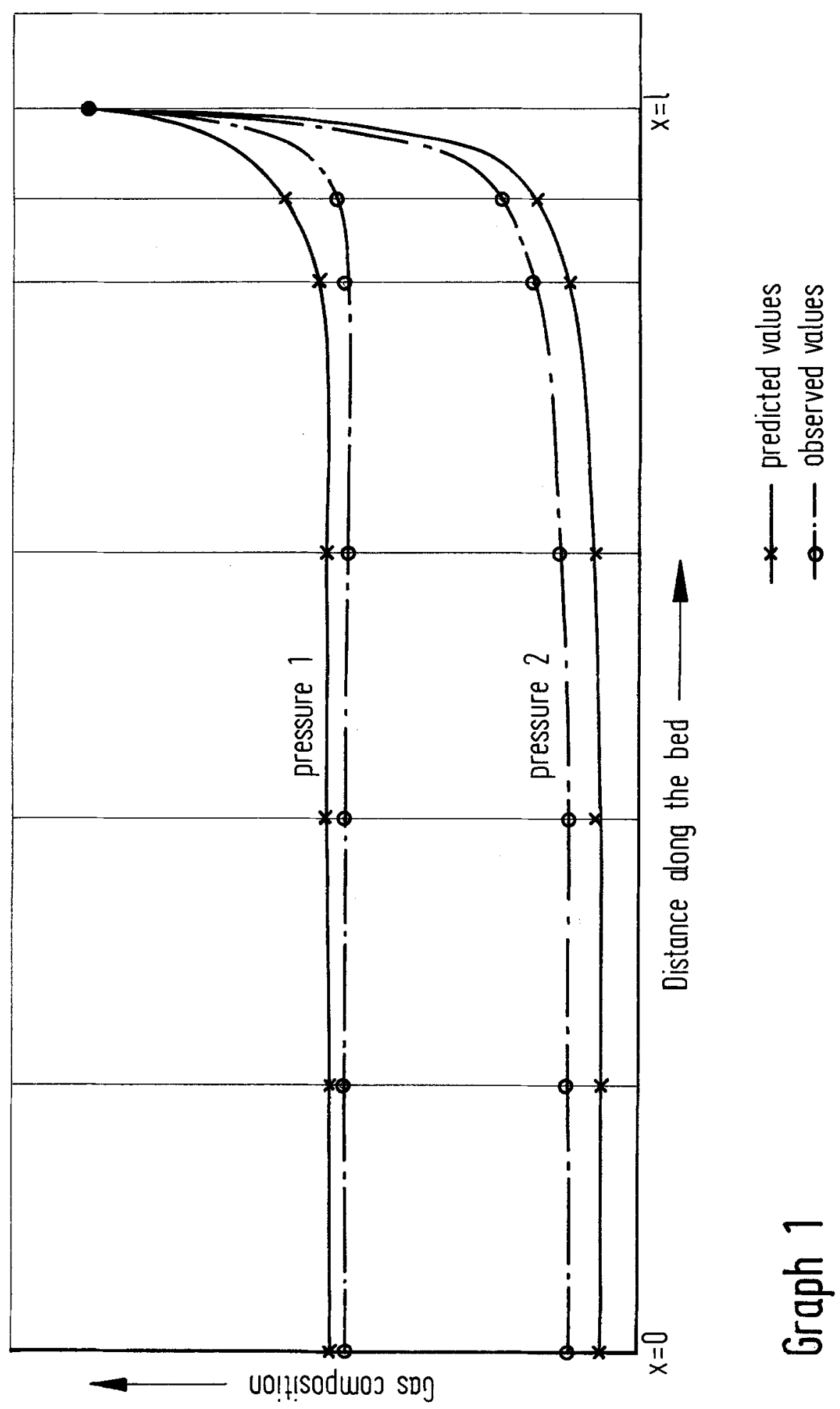




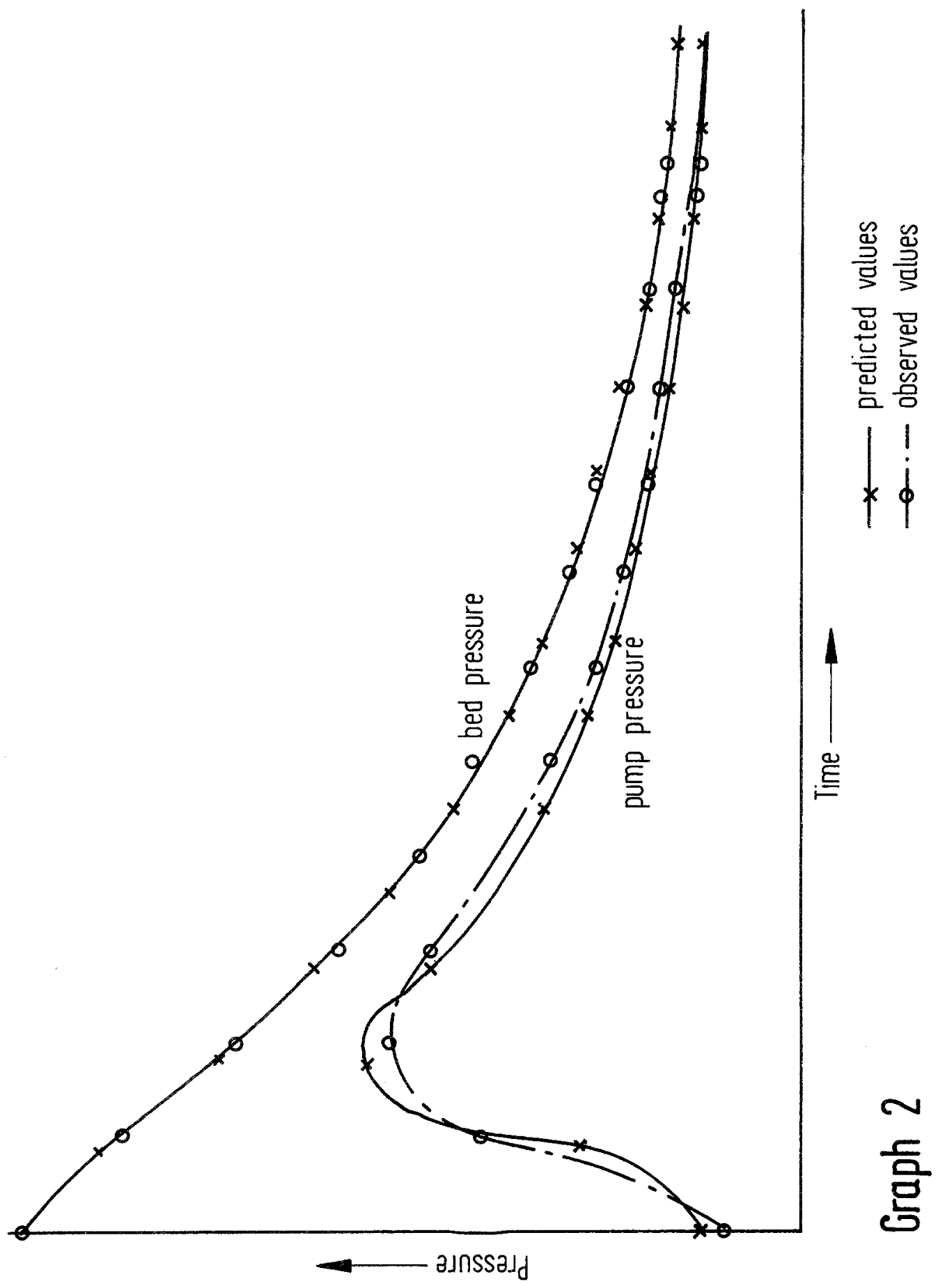




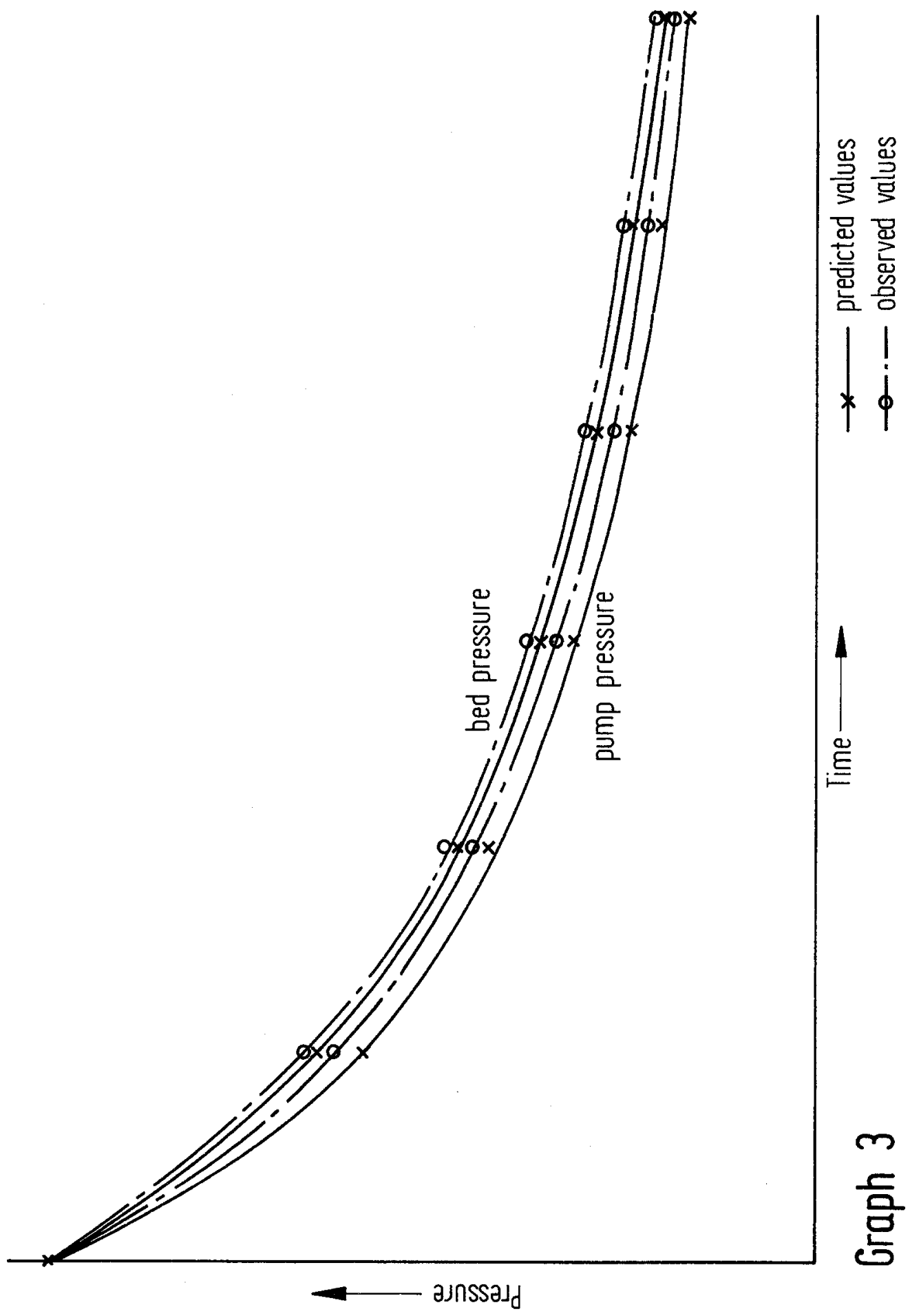




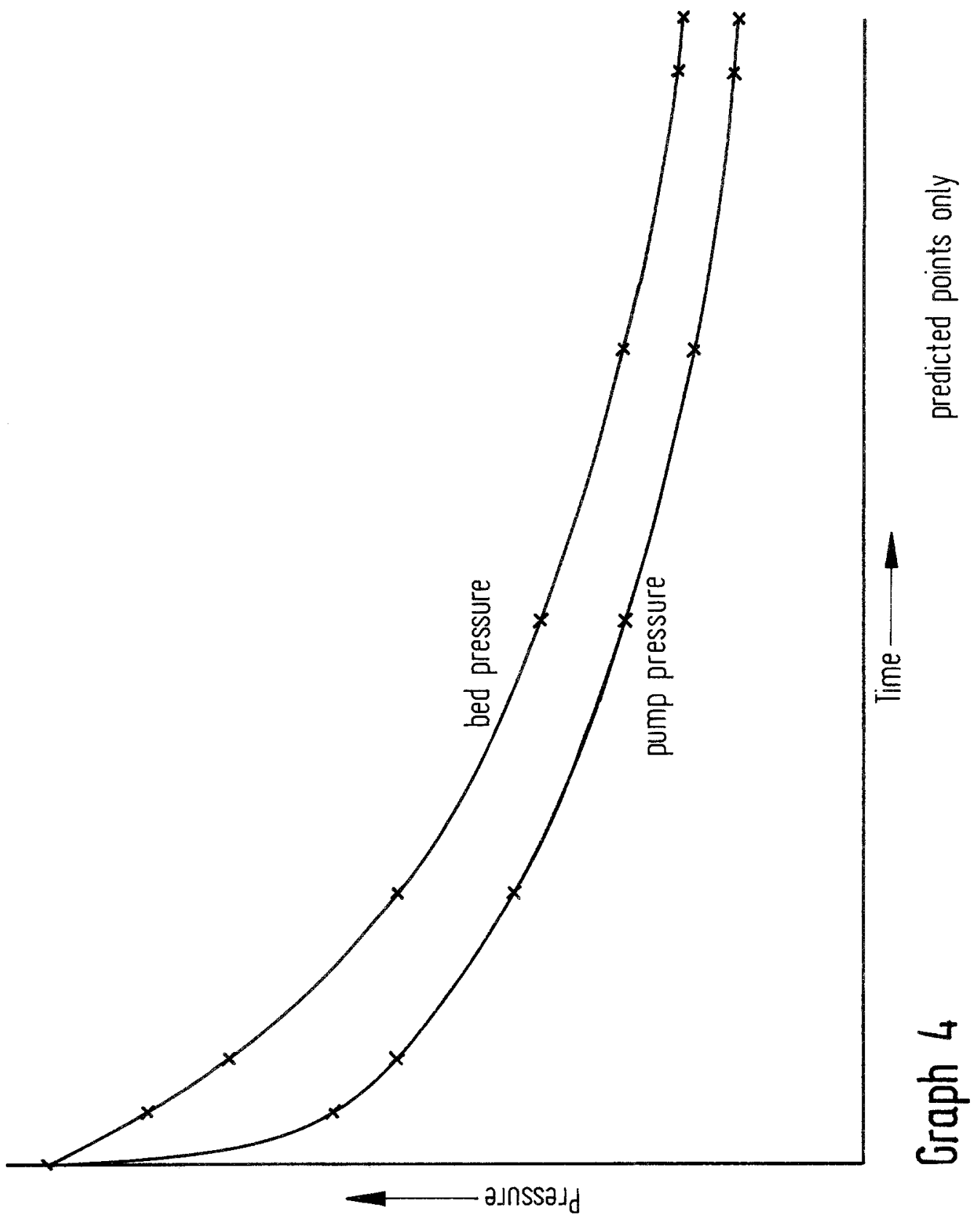

